\title{
Nordiques
}

38 | 2019

Le petit héros scandinave

\section{Pippi Långstrump et la France : une conquête par étapes}

\section{Cyrille François}

\section{(2) OpenEdition \\ Journals}

Édition électronique

URL : http://journals.openedition.org/nordiques/290

DOI : $10.4000 /$ nordiques. 290

ISSN : $2777-8479$

Éditeur :

Association Norden, Bibliothèque de Caen la mer

Édition imprimée

Date de publication : 1 novembre 2019

Pagination : 44-57

ISBN : 979-1-0959140-4-4

ISSN : 1761-7677

Référence électronique

Cyrille François, "Pippi Långstrump et la France : une conquête par étapes », Nordiques [En ligne], 38 |

2019, mis en ligne le 31 octobre 2020, consulté le 14 mars 2021. URL : http://

journals.openedition.org/nordiques/290; DOI : https://doi.org/10.4000/nordiques.290

Nordiques 


\section{Pippi Långstrump et la France : une conquête par étapes}

Cyrille François*

\section{RÉSUMÉ}

Au début des années 1990, la linguiste suédoise Christina Heldner lance un pavé dans la mare : la traduction française de Pippi Långstrump par Marie Loewegren comporte de nombreux défauts qui expliqueraient en partie le manque de succès en France du roman d'Astrid Lindgren. Parvenus aux oreilles de l'auteure, les propos de Heldner contribuent à faire paraître une nouvelle traduction des aventures de Fifi Brindacier, après de nombreux échanges entre Astrid Lindgren et les éditions Hachette. L'histoire éditoriale de Pippi Långstrump en France est toutefois plus complexe encore, car la traduction de Marie Loewegren cache en fait deux éditions, dont la seconde est largement remaniée par l'éditeur afin de conformer le texte à la nouvelle ligne éditoriale de la Bibliothèque rose. Cet article vise à comprendre les différentes étapes de la conquête de Pippi Längstrump en France.

\section{ABSTRACT}

In the early 1990s, Swedish linguist Christina Heldner dropped a bombshell: Marie Loewegren's French translation of Pippi Långstrump had many flaws that could in part explain the lack of success in France of Astrid Lindgren's novel. Heldner's comments, which came to the author's attention, contributed to the publication of a new translation of Pippi Longstocking's adventures, after numerous exchanges between Astrid Lindgren and Hachette publishing. The editorial history of Pippi Långstrump in France is even more complex, however, because Marie Loewegren's translation actually hides two editions, the second of which is largely reworked by the publisher to conform the text to the new editorial line of the Bibliotheque rose. This article aims to explore the different stages of Pippi Laingstrump's conquest of France.

\footnotetext{
* Cyrille François est maître d'enseignement et de recherche à l'université de Lausanne. Ses recherches portent sur les stratégies narratives des contes de Perrault, Grimm et Andersen, ainsi que sur les questions de traduction et d'adaptation de textes littéraires. Il a écrit Les Voix des contes : stratégies narratives et projets discursifs des contes de Perrault, Grimm et Andersen (PUBP, 2017) et édité une collection bilingue de contes et histoires choisis d'Andersen (Classiques Garnier, 2017). Il est également coéditeur d'un numéro de Deshima sur « Le(s) Nord de Xavier Marmier » (2018).
} 
Qualifié de "classique mondial de la littérature de jeunesse " dans le texte de présentation de l'édition Le Livre de Poche (2007), Fifi Brindacier [Pippi Langstrump], d'Astrid Lindgren, raconte les aventures de cette petite fille forte et facétieuse connue du plus grand nombre, et présente tant en librairie qu'à la télévision, sous différentes formes ${ }^{1}$. Le même texte de présentation annonce les raisons d'un tel succès :

C'est que, dans l'univers bien sage de ce qu'on appelait alors la "littérature enfantine ", le personnage si neuf et si exceptionnel qu'était Fifi Brindacier, libre, primesautier, imprévisible, faisait irruption avec une joyeuse hardiesse. Ce fut un coup de vent émoustillant.

Décrivant la réception de cette œuvre en Suède, Lena Kåreland précise quant à elle :

Fifi Brindacier, créée par Astrid Lindgren, est indiscutablement le premier personnage véritablement moderne de la littérature enfantine suédoise, Fifi s'efforce constamment de rester libre, par rapport aux parents aussi bien que vis-à-vis des conventions. Elle incarne le Moi sans entraves, sans masques, sans hypocrisie, libre des déformations sociales. Fifi personnifie le chaos fertile. Elle symbolise la vie authentique, le vrai et le créatif. L'aspect provocateur du personnage reste vivace, même à l'heure du postmodernisme. ${ }^{2}$

Ces caractéristiques sont souvent convoquées pour justifier le succès des livres d'Astrid Lindgren dans le monde entier, mais est-ce que cette Pippi Långstrump indépendante, anticonformiste, libre, créative et provocatrice correspond réellement à la Fifi Brindacier " primesautière » de la présentation des éditions Le livre de Poche ? Et est-ce vraiment elle qui a conquis la France ?

Sur le plan des ventes, Fifi Brindacier représente un véritable succès, Cécile Térouanne, directrice de Hachette Romans/Livre de Poche Jeunesse, confirmant que le livre est un best-seller ${ }^{3}$. Il semblerait néanmoins que le succès ait été plus tardif que dans d'autres pays. Une recherche dans le catalogue de la BNF indique que le phénomène s'est accentué après les années 2000 : sur les 43 occurrences de "Brindacier ", seulement 4 sont antérieures à $1995^{4}$. Valérie Landais-Alfvén affirme ainsi en 2001 que les ventes sont beaucoup moins importantes en France

1 L'édition originale est parue en trois volumes chez Rabén \& Sjögren, à Stockholm : Pippi Långstrump (1945), Pippi Långstrump går ombord (1946), Pippi Långstrump i Söderhavet (1948). Ils sont réunis en un seul volume (avec quelques différences) dans Astrid Lindgren, Boken om Pippi Långstrump, Stockholm, Rabén \& Sjögren, 1962.

2 Lena Kåreland, «Le modernisme dans la littérature enfantine en Suède. Quelques lignes directrices », Germanica, $\mathrm{n}^{\circ} 12,1993$, p. 130.

3 Les chiffres exacts ne peuvent cependant pas être communiqués. Voir aussi son article : Cécile Térouanne, « Hachette et l'édition d'Astrid Lindgren en France », La revue des livres pour enfants, ${ }^{\circ} 238$, 2007, p. 130-134.

4 Critères de recherche : textes imprimés, auteur=astrid lindgren, langue=français. On observe la même tendance pour une recherche "Fifi " (ce qui permet de prendre en compte le deuxième volume, souvent intitulé Fifi princesse) : 48 notices, dont seulement 7 avant 1995. 
que dans les pays voisins et que les jeunes Français ne connaissent pas aussi bien le personnage de Fifi Brindacier ${ }^{5}$. En 1981, Bernard Epin utilisait d'ailleurs une modalisation révélatrice lorsqu'il commentait l'œuvre d'Astrid Lindgren, "plus ou moins reconnue comme un classique international $"^{6}$. Le succès en librairie n'est peut-être pas aussi important que chez nos voisins allemands, italiens ou néerlandais, mais Pippi Långstrump bénéficiait sans doute au moins d'un succès d'estime, comme le confirme Bernard Epin quand il enjoint à " relire Fifi Brindacier en le dépouillant de ses auréoles ${ }^{7}$.

Le succès tardif des ventes pourrait être lié à la nouvelle traduction proposée par Alain Gnaedig en $1995^{\circ}$, à la sortie d'un dessin animé en 1998, voire à l'évolution du marché de la littérature de jeunesse. Les volumes originaux sont en effet accompagnés depuis ces années-là de plusieurs albums autour de Fifi Brindacier, traductions tardives des albums publiés en Suède dans les années 1969-1971. Peut-être est-ce aussi lié à l'évolution de l'image même de Fifi Brindacier, dont la modernité peut plus facilement être acceptée en France que dans les années 1950. On peut ainsi se demander si c'est vraiment une fillette anticonformiste qui avait conquis le public français lorsqu'on lit le compte rendu que faisait le jésuite André Ravier en 1962 dans la revue catholique Études:

Fifi Brindacier, par Astrid Lindgren, dont l'humour suédois et les images griffées (de Noëlle Lavaivre) déconcertent d'abord plus d'un lecteur, s'avère en définitive un livre très amusant, riche de cœur autant que de fantaisie?.

Nous mettrons en relation le succès du livre avec son histoire éditoriale française, afin de montrer que la conquête de la France par Pippi Långstrump a connu plusieurs étapes. Des commentateurs ont déjà souligné, à la suite de Christina Heldner, que la première traduction française, par Marie Loewegren, opérait une sorte de censure en proposant un livre beaucoup moins subversif que l'original et dont le style était plus formel ${ }^{10}$. Plusieurs chapitres n'avaient par ailleurs pas été conservés. La nouvelle traduction, souhaitée par Astrid Lindgren elle-même qui est intervenue avec conviction auprès de l'éditeur, a vu le jour en 1995. Réalisée par Alain Gnaedig, elle présente un roman plus proche du texte original.

5 Valérie Landais-Alfvén, Fifi Brindacier d'Astrid Lindgren : étude d'une métamorphose et d'une renaissance, Mémoire de maîtrise sous la direction d'Isabelle Nières-Chevrel, université de Rennes II, 2001, p. 103-105.

6 Bernard Epin, "Les hits-parades d'Astrid Lindgren ", La revue des livres pour enfants, ${ }^{\circ}$ 79, juin 1981 , p. 14 .

7 Ibid., p. 15.

8 Dans ce travail, je citerai l'édition de 2007 qui réunit les trois volumes de la traduction : Astrid Lindgren, Fifi Brindacier, trad. Alain Gnaedig, ill. Ingrid Vang Nyman, Paris, Hachette jeunesse (Le Livre de Poche), 2007.

9 André Ravier, «Livres d'étrennes ", Études, décembre 1962, p. 423.

10 Les recherches de Christian Heldner (voir la note 16 ci-dessous) sont reprises dans de nombreux livres sur la littérature de jeunesse et dans de nombreux articles. Voir par exemple Hans Hartje, "Pippi Långstrump ou l'enfant terrible...ment variable », Publije, vol. 1, 2010, p. 1-8. 
Ce cas n'est pas inédit dans le domaine de la traduction et cela correspond au phénomène décrit par "l'hypothèse de la retraduction ", présentée ainsi par Paul Bensimon dans le numéro de 1990 de la revue Palimpsestes:

Il existe des différences essentielles entre les premières traductions, qui sont des introductions, et les retraductions. La première traduction procède souvent — a souvent procédé — à une naturalisation de l'œuvre étrangère ; elle tend à réduire l'altérité de cette œuvre afin de mieux l'intégrer à une culture autre. Elle s'apparente fréquemment - s'est fréquemment apparentée - à l'adaptation en ce qu'elle est peu respectueuse des formes textuelles de l'original. La première traduction vise généralement à acclimater l'œuvre étrangère en la soumettant à des impératifs socioculturels qui privilégient le destinataire de l'œuvre traduite. Ainsi, par exemple, les traducteurs qui ont opéré des coupures dans le texte d'origine ont-ils eu pour préoccupation maitresse d'assurer une meilleure réception auprès de leur public. [...] Les retraductions diffèrent, par plusieurs traits fondamentaux, des premières traductions. La première traduction ayant déjà introduit l'œuvre étrangère, le retraducteur ne cherche plus à atténuer la distance entre les deux cultures ; il ne refuse pas le dépaysement culturel : mieux, il s'efforce de le créer. Après le laps de temps plus ou moins grand qui s'est écoulé depuis la traduction initiale, le lecteur se trouve à même de recevoir, de percevoir l'œuvre dans son irréductible étrangeté, son " exotisme ». La retraduction est généralement plus attentive que la traduction-introduction, que la traduction-acclimatation, à la lettre du texte source, à son relief linguistique et stylistique, à sa singularitén ${ }^{11}$.

La première traduction de Pippi Långstrump représenterait ainsi, selon cette hypothèse de la retraduction, une étape nécessaire d'adaptation aux « impératifs socioculturels » et éditoriaux du champ français. Annelie Jarl Ireman a sans doute raison quand elle écrit que la France "n'était tout simplement pas prête pour Pippi Långstrump à cette époque ", tant le livre était subversif, et que c'est grâce à cette traduction problématique qu'il a connu le succès ${ }^{12}$.

Cependant, le dossier est bien plus complexe qu'il n'y paraît, car les deux traductions de Loewegren et de Gnaedig cachent un autre livre. La première traduction a en effet connu une deuxième édition fortement remaniée, en 1962, vraisemblablement par l'éditeur ${ }^{13}$. Or, cette traduction remaniée, qui coïncide

11 Paul Bensimon, «Présentation », Palimpsestes, n 4, 1990, \$1-3, disponible sur http://palimpsestes. revues.org/598 [consulté le 18/12/2016].

12 Annelie Jarl Ireman, "Svensk barn- och ungdomslitteratur i Frankrike - från Nils till Semlan : Ställning, urval och anpassning », in Mickaëlle Cedergren et Sylvain Briens (éds.), Médiations interculturelles entre la France et la Suède. Trajectoires et circulations de 1945 à nos jours, Stockholm University Press, 2015, p. 223.

13 Chaque édition comporte en fait deux volumes, dont voici les références exactes : Astrid Lindgren, Mademoiselle Brindacier, trad. Marie Loewegren, ill. Mixi-Bérel, Hachette, 1951 ; Astrid Lindgren, La Princesse de Couricoura, trad. Marie Loewegren, ill. Marianne Clouzot, Hachette, 1953 ; Astrid Lindgren, Fif $\boldsymbol{i}$ Brindacier, trad. Marie Loewegren, ill. Noëlle Lavaivre, Hachette, 1962 ; Astrid Lindgren, Fif princesse, trad. Marie Loewegren, ill. Noëlle Lavaivre, Hachette, 1963. Dans la suite de l'article, on s'y référera en mentionnant l'année d'édition. 
avec un tirage bien plus important du livre, dû à la modernisation des éditions Hachette ${ }^{14}$, est celle qui a popularisé Fifi Brindacier. Les différences entre l'édition de 1951 et celle de 1962 ont peu été étudiées, même si elles sont mentionnées dans quelques articles et mémoires de maîtrise ${ }^{15}$. Il s'agit ainsi de mener une véritable analyse comparative afin de tenter, d'une part, de comprendre en quoi consistent ces différences, et d'autre part, comment elles ont été opérées et ce que l'on peut en conclure sur la réception de Pippi Långstrump en France et sa conquête du public français.

Nous nous pencherons sur la genèse de la retraduction de 1995, avant d'aborder la réédition de la première traduction, dans un premier temps par une comparaison des textes, puis dans un second temps par une étude sur la mutation de la Bibliothèque rose qui permet de mieux comprendre le processus de transformation du texte.

\section{LA GENÈSE DE LA RETRADUCTION}

Au début des années 1990, une linguiste suédoise, Christina Heldner, suggère que le manque de succès de Fifi Brindacier en France résulte en grande partie des nombreux défauts de la traduction française ${ }^{16}$. Ses travaux créent une certaine agitation dans les milieux littéraires et le propos parvient à Astrid Lindgren, qui, après plusieurs échanges - parfois cocasses - avec les éditions Hachette, parvient à faire publier les aventures de Pippi Långstrump dans une nouvelle traduction par Alain Gnaedig en 1995. L'histoire est bien résumée dans l'un des derniers articles de Heldner ${ }^{17}$. Cette partie du dossier étant assez bien connue, je me contenterai de citer quelques éléments de la critique de la linguiste.

14 Sophie Heywood, "Modernising and moralising: Hachette's mass-market fiction series for children, 1950s-1960s ». Strene, $\mathrm{n}^{\circ} 11,2016, \$ 11$, disponible sur http://strenae.revues.org/1640 [consulté le 10/11/2016].

15 Voir par exemple Valérie Landais-Alfvén, op. cit.; Claire Dupont, La littérature de jeunesse suédoise en traduction française: une étude de Fifi Brindacier, de Jan, mon ami et de Pourquoi mon père porte de grandes chaussures, Mémoire de Master 1 sous la direction d'Annelie Jarl Ireman, université de Caen, 2013 ; Annelie Jarl Ireman, op. cit. ; Sophie Heywood, " Pippi Longstocking, Juvenile Delinquent? Hachette, Self-Censorship and the Moral reconstruction of Postwar France ", Itinéraires, n 2, 2015, p. 1-12.

16 Christina Heldner, "Pippi Långstrump i fransk tvångströja ", in Karl Johan Danell, Gunnar Persson et Astrid Stedje (éds.), Språket som kulturspegel, Stockholm, Almqvist \& Wiksell International, 1991, p. 63-74. Une traduction française de l'article paraît en 1992 (Christina Heldner, «Une anarchiste en camisole de force. Fifi Brindacier ou la métamorphose française de Pippi Langstrump ", La revue des livres pour enfants, $\mathrm{n}^{\circ} 145,1992$, p. 65-71), puis Heldner publie plusieurs articles consacrés à cette question dans les années qui suivent.

17 Christina Heldner, «Hur Pippi Långstrump slapp ur sin franska tvångströja », Barnboken, Svenska barnboksinstitutets tidskrift, $\mathrm{n}^{\circ}$ 1, 2004, p. 11-21. 
Premièrement, les trois volumes suédois sont concentrés en deux volumes. Quatre chapitres sont tout bonnement supprimés, et ceux qui sont conservés n'en sortent pas indemnes : la traduction supprime les aventures rocambolesques de Pippi et les passages qui présentent des adultes désagréables ou insolents contre lesquels Pippi se défend (et dont elle se moque souvent avec gentillesse). Seule exception : lorsque l'héroïne reconnaît sa faute et s'en excuse, le passage peut être maintenu. Par extension, d'autres cas sont conservés après l'ajout de remords en français (bien qu'inexistants en suédois). On en trouve un exemple lorsque Pippi est aux prises avec une dame de charité, Mademoiselle Fleuriche (Fröken Rosenblom en suédois) :

- Si tu ne te mets pas immédiatement au coin, dit Mademoiselle Fleuriche, je connais une petite fille qui va bientôt recevoir une bonne fessée !

- Ne te mets pas en colère, pria Fifi. Je te demande pardon, si je t’ai fait de la peine. Je suis insupportable, mais, tu sais, quand on vit toujours tout seul, on finit par être pas comme tout le monde..."

$\mathrm{M}^{\mathrm{Il}}$ Fleuriche était tout émue. Elle alla embrasser Fifi, qui alors s'en fut vers le groupe des enfants en pénitence ${ }^{18}$.

La réponse de Pippi et le dénouement heureux de cette entrevue n'ont rien à voir avec le texte original, dans lequel l'héroïne ne comprend pas ce type de menace énoncée sous forme de périphrase. Pippi répond ainsi au premier degré en faisant référence à une autre petite fille :

- Om du inte genast går och ställer dej där borta och skäms, sa fröken Rosenblom, så vet jag en liten flicka som snart fär ordentligt med smäll.

- Stackars unge, sa Pippi. Var är hon? Skicka henne till mej, så ska jag forrsvara henne. Anteckna det!

Och så gick Pippi och ställde sig bland barnen som skulle skämmas ${ }^{19}$.

Plus que l'insolence de Pippi, le texte original mettait en évidence le chantage des adultes et l'utilisation de formules équivoques plutôt que d'un langage direct.

Deuxièmement, le caractère oral de l'œuvre originale est altéré. Les marques de présence de la narratrice dans le récit sont supprimées ${ }^{20}$, de même que les répétitions ; le langage concret, les jeux de mots et les néologismes sont fréquemment remplacés par un langage abstrait et, globalement, plus « écrit ». En fin de compte, le lecteur n'a plus vraiment l'impression qu'on lui raconte l'histoire.

18 Astrid Lindgren, op. cit., 1953, p. 153.

19 Astrid Lindgren, Boken om Pippi Långstrump, p. 249. On pourrait traduire ainsi la réponse de Pippi : « Pauvre petite, dit Fifi. Où est-elle ? Envoie-la vers moi, je pourrai la protéger. »

$20 \mathrm{La}$ traduction supprime par exemple ce commentaire indiqué en italique (que je traduis entre crochets) : « Det var den märkvärdigaste flicka Tommy och Annika hade sett, och det var Pippi Långstrump, som gick ut på morgonpromenad. Så här såg hon ut: [...] " (Astrid Lindgren, Boken om Pippi Långstrump, p. 11) ; "C'était la petite fille la plus singulière que Tommy et Annika eussent jamais vue. Fifi Brindacier partait faire sa promenade matinale. [Voici de quoi elle avait l'air :] (Astrid Lindgren, 1962, p. 9). 
Un court extrait suffit à se faire une idée de l'étendue des modifications :

En gång i tiden hade Pippi haft en pappa, som hon tyckte förfärligt mycket om, ja, hon hade förstås haft en mamma ocksä, men det var så länge sedan, så det kom hon inte alls ihåg. Mamman hade dött, när Pippi bara var en liten, liten unge, som låg $\boldsymbol{i}$ vaggan och skrek så förskräckligt, att ingen kunde vara $i$ närheten. Pippi trodde, att hennes mamma nu satt uppe $\boldsymbol{i}$ himlen och kikade ner på sin flicka genom ett litet hål, och Pippi brukade ofta vinka upp till henne och säja:

- Var inte ängslig! Jag klarar mej alltid! ${ }^{21}$

Fifi avait eu autrefois un papa qu'elle aimait de tout son cour et, bien sûr, une maman. Sa maman était morte alors que Fifi n'était encore qu’un bébé. Fifi s'imaginait souvent que sa maman la regardait par une échappée entre les nuages. Et, parfois, il lui arrivait de lever la tête pour dire : "Ne t’inquiète pas, maman ! Je me débrouillerai toujours ! "22

Changement de registre (« förfärligt mycket »/« terriblement » remplacé par " de tout son cour »), suppression d'interjections ( « ja »/ « oui »), de répétitions d'adjectifs (" liten, liten »/ " petit, petit »), remplacement d'une expression concrète ( litet hål »/ " petit trou ») par une expression abstraite ( échappée entre les nuages ") ; la traduction présente de nombreux changements stylistiques en plus des suppressions de propositions : « men det var så länge sedan, så det kom hon inte alls ihåg " (mais c'était il y a si longtemps qu'elle ne s'en souvenait plus du tout) ; " som låg i vaggan och skrek så förskräckligt, att ingen kunde vara i närheten " (qui gisait dans son berceau et criait si terriblement que personne ne pouvait rester dans les parages).

Dans la nouvelle traduction d'Alain Gnaedig, ce passage, de même que les autres exemples que je viens de citer, est plus proche du texte original :

Fifi avait eu autrefois un papa qu'elle adorait et, bien sûr, elle avait eu aussi une maman. Mais c'était il y a si longtemps qu'elle ne s'en souvenait plus du tout. La maman de Fifi était morte quand celle-ci n'était qu'un tout petit bébé qui braillait si fort dans sa poussette que personne n'arrivait à rester à côté d'elle. Fifi pensait que sa maman se trouvait au ciel et qu'elle l'observait par un petit trou entre les nuages. Fifi lui faisait souvent un petit signe et lui disait : / - Ne t'inquiète pas! Je me débrouillerai toujours !23

Bien que des modifications soient apportées (nous en indiquons quelques-unes en italique), Gnaedig conserve tous les éléments du texte suédois et cherche à mieux rendre sa dimension orale.

21 Astrid Lindgren, Boken om Pippi Långstrump, op. cit., p. 7-8.

22 Astrid Lindgren, op. cit., 1962, p. 6.

23 Astrid Lindgren, op. cit., 2007, p. 10. 
Heldner résume les problèmes de la traduction de Marie Loewegren en précisant que la traductrice a un "souci constant soit d'atténuer ou de censurer tous les traits de l'œuvre d'Astrid Lindgren qui vont à l'encontre d'une éducation traditionaliste, soit de conformer son récit et son style à un certain idéal littéraire extrêmement conventionnel $»^{24}$. Ceci est d'autant plus dérangeant que le français neutralise ainsi des effets précisément développés par Lindgren entre son manuscrit et le livre édité en suédois ${ }^{25}$. Relevons toutefois que l'édition française de 1962 suit parfois le type de modifications qu'Astrid Lindgren a elle-même opérées. L'édition suédoise présente par exemple un passage à l'école où Pippi est triste et regrette son comportement, alors que ce regret est absent du manuscrit ${ }^{26}$. L'auteure a donc parfois moralisé son récit pour le conformer aux attentes de l'éditeur ; la traduction française poursuit quant à elle ce travail de moralisation pour conformer le récit au champ français fortement marqué par la loi de 1949 sur les publications destinées à la jeunesse qui a créé une atmosphère d'autocensure dans le monde de l'édition.

\section{UNE TRADUCTION RÉÉDITÉE}

La comparaison est éclairante et les travaux de Heldner, qui décrivent très bien l'édition de 1962-63, ont joué un rôle déterminant pour la réception de Pippi Långstrump en français en incitant Hachette à publier une nouvelle traduction. La linguiste a par ailleurs une bonne intuition quand elle fait le lien entre les modifications et le contexte culturel de l'époque, notamment avec la loi de 1949 Dans ce contexte, il n'est pas étonnant de lire cet avertissement inséré au début de la traduction de 1951:

Fifi Brindacier est très populaire en Suède. Ce personnage de fillette, douée d'une force physique extraordinaire, n'est pas emprunté à la vie réelle. Il ne doit son existence qu'à l'imagination de l'auteur.

Il semble appliquer à la lettre les principes du « Compte rendu des travaux de la commission de surveillance et de contrôle des publications destinées à l'enfance et à l'adolescence au cours de l'année 1950 » qui invitent à " éviter l'excès d'invraisemblance $[\ldots]$ risquant de faire confondre le réel et l'imaginaire $»^{27}$.

S'il est nécessaire aujourd'hui de remettre l'ouvrage entrepris par Heldner sur le métier, c'est parce que la linguiste a négligé une étape importante de la réception française de Pippi Långstrump: elle travaille sur la traduction de Marie Loewegren

24 Christina Heldner, "Une anarchiste en camisole de force », op. cit., p. 67.

25 Comparant le manuscrit au livre, Ulla Lundqvist écrit, exemples à l'appui, que le style de l'édition est plus clair et que la langue "se rapproche plus du niveau de compréhension des enfants " (Astrid Lindgren, Ur-Pippi. Originalmanus, Stockholm, Rabén \& Sjögren, 2007, p. 147).

26 Ibid., p. 132-133.

27 Cité dans Sophie Heywood, « Pippi Longstocking », op. cit., note 15. 
dans l'édition Livre de Poche Jeunesse de 1990 et non sur l'édition originale de sa traduction. Une note de bas de page de son article indique l'existence d'une édition antérieure, mais sans la décrire. Or, comme je l'ai annoncé, le texte de la traductrice a été largement remanié. La plupart des défauts que relève Heldner sont d'ailleurs absents de la première édition de 1951. Il faut ainsi entreprendre un travail philologique pour historiciser les édition $s^{28}$ et mieux prendre en compte le travail des éditeurs. Heldner elle-même revoit en partie sa position dans son article de 2004 après avoir discuté avec une auteure publiée dans les années 1960 dans La Bibliothèque rose qui décrit les nombreuses révisions imposées par l'éditeur. La linguiste précise alors que Marie Loewegren a dû « être soumise aux mêmes types de pression ${ }^{29}$.

Peu d'études proposent la comparaison des éditions de 1951/1953 et de 1962/1963, car la plupart des articles sur la traduction française de Pippi Långstrump reprennent les travaux de Heldner. Même lorsque les éditions sont bien décrites, comme dans le mémoire de Valérie Landais-Alfvén, elles ne sont pas réellement comparées : partant du principe que l'édition de 1962 est une sorte de résumé de celle de 1951, Landais-Alfvén analyse les deux textes ensemble pour les opposer à la nouvelle traduction d'Alain Gnaedig.

Or, il faut le répéter, la plupart des défauts que relève Heldner sont absents de la première édition. Aucune des aventures rocambolesques n'est supprimée, et Fifi Brindacier présente la même irrévérence que Pippi Långstrump. En témoigne ce passage entre l'héroöne et la maîtresse d'école largement adapté dans la deuxième édition :

Non, ma petite bonne femme, tu vas trop loin, dit Fifi. Tu m'as dit tout à l'heure que c'est 7 et 5 qui faisaient 12. Faut tout de même un peu d'ordre, dans une école. D'ailleurs, si ces bêtises-là t'amusent tellement, pourquoi ne pas t'asseoir dans un coin ; tu ferais tes petits calculs tout à ton aise, et nous, nous pourrions jouer tranquilles ? Allons bon, voilà que je te dis encore "tu". Peux-tu me pardonner cette fois encore ?... ${ }^{30}$

Cette fois, chère mademoiselle, je ne comprends plus du tout, dit Fifi. Tu viens de me dire que c'est 7 et 5 qui font 12 . Allons bon, voilà que je te dis toujours "tu". Peux-tu me pardonner cette fois encore ?....

28 Souvent négligée, la genèse post-éditoriale des œuvres révèle souvent des surprises. Voir à ce sujet le numéro 44 de Genesis, coordonné Rudolf Mahrer : «Après le texte : de la réécriture après publication » (2017).

29 Christina Heldner, " Hur Pippi Långstrump slapp ur... ", op. cit., p. 13.

30 Astrid Lindgren, op. cit., 1951, p. 51.

31 Astrid Lindgren, op. cit., 1962, p. 37. 
On peut par endroits relever quelques changements où Loewegren atténue la charge contre les adultes ${ }^{32}$, mais on ne peut pas parler de censure dans l'édition de 1951 comme le fait Heldner pour celle de 1962. Seule la perspective enfantine du récit fait réellement défaut dans l'édition de 1951 : "un papa et une maman " devient ainsi " un homme et une femme" (p. 11) et la voix narrative prend parfois la perspective d'adultes, d'éducateurs, par l'ajout de commentaires sur les enfants qui doivent "être grondés quand ils sont désobéissants " ${ }^{33}$.

Le seul véritable contre-exemple concerne l'un des passages cité plus haut, avec la dame de charité, car les regrets de Pippi sont présents en 1951. Il y a donc déjà une forme de moralisme dans la première édition de la traduction. Mais là aussi le dossier est bien plus complexe qu'il n'y paraît, car une recherche à l'Institut mémoires de l'édition contemporaine (IMEC) révèle que les excuses de Fifi ont été ajoutées lors du travail éditorial. L'IMEC possède un tapuscrit de ce volume retouché par l'éditeur. On y lit une réponse qui correspond au texte suédois : " "Pauvre petite!" dit Fifi "Où est-elle donc ? Envoie-là moi, je saurai bien la défendre" ». Cette réponse est corrigée à la main et remplacée par une formulation très proche de celle qui sera conservée dans l'édition de $1953^{34}$.

Du point de vue stylistique, l'édition de 1951/1953 perd déjà une partie de l'oralité du texte suédois, mais elle est néanmoins écrite dans un registre moins soutenu que l'édition de 1962, comme en témoignent ces quelques exemples modifiés dans la deuxième édition ${ }^{35}$ :

- Négation uniformisée : «T’en fais pas, maman !» $\rightarrow$ «Ne t'inquiète pas, maman!»

- Répétition supprimée : «Le papa de Fifi avait acheté la vieille maison, il y avait très, très longtemps "

- Suppression du connecteur " et " en début de phrase ou de paragraphe

- Phrase nominale supprimée : "Ils l'empoignèrent et essayèrent de la soulever. /Peine perdue. " $\rightarrow$ "Ils l'empoignèrent et essayèrent de la soulever. /Une fois encore, ce fut peine perdue. "

- Suppression de pataquès : " Je vous suivrai n’importe où, dit Fifi, mais ké-ksé-ksa, un cirque ?" $\rightarrow$ "Je vous suivrai n'importe où ! s'écria Fifi. Mais qu'est-ce qu'un cirque?"

L'étude du tapuscrit révèle là encore qu'une grande partie de la normalisation du style de la première édition est déjà due à l'éditeur. En plus des corrections

32 Les gendarmes sont par exemple décrits de manière moins ridicule quand ils naviguent sur le toit de la maison de Pippi, dans le chapitre 3.

33 Astrid Lindgren, op. cit., 1951, p. 36.

34 Tapuscrit de La Princesse de Couricoura, IMEC, HAC 6926 (ex S14 C162 B1), f 74.

35 Astrid Lindgren, op. cit., 1951, p. 8, 9, 93, 88 ; Astrid Lindgren, op. cit., 1962, p. 6, 7, 8, 72 et 67. 
orthographiques, on y voit plusieurs gestes de normalisation de la langue : " plusieurs » est remplacé par " davantage »; " par contre » par " en revanche »; le subjonctif imparfait est ajouté partout où le demande le principe de concordance des temps ; le lexique est plus élaboré (" dire " $\rightarrow$ " répliquer », " annoncer "; " très peur $» \rightarrow$ "grand peur $»)$.

Ces derniers exemples invitent à se pencher sur le type et l'étendue du travail éditorial, qui commence dès la première édition, mais qui se révèle nettement plus important pour la deuxième édition, en 1962/1963. On peut en conclure que la normalisation de la langue n'est pas imputable à la traductrice, mais à l'éditeur.

\section{LA MUTATION DE LA BibliothÈQUe ROSE}

La traduction française suit en fait la même évolution que la Bibliothèque rose elle-même, qui a subi de nombreuses transformations dans les années 1950-1960, autour de son directeur, Louis Mirman, en poste depuis $1953^{36}$. La première édition de Mademoiselle Brindacier est parue dans la Bibliothèque rose " ancienne version ". La mue de la collection s'opère par la suite avec une politique éditoriale très claire, tant du point de vue idéologique que formel. Afin de conserver sa position prédominante, Hachette développe de nouvelles techniques de production dont un des effets est de standardiser les volumes. L'une des caractéristiques de cette nouvelle formule est la couverture cartonnée en couleur qui remplace les cartonnages toilés et qui permet d'imprimer la couverture directement sur le livre $^{37}$. Le nombre de pages par volume devient également moins flexible.

La traduction de Loewegren subit en 1962 un processus de transformation visant à conformer le texte à la nouvelle ligne éditoriale de la Bibliothèque rose. Plus que de simples retouches, il s'agit d'un complet remodelage. La maison d'édition donne en effet à l'époque des consignes très précises aux auteurs et aux traducteurs, les notes de lecture se multiplient, les corrections s'intensifient, et l'on dénote une bureaucratisation extrême. Cette ligne éditoriale se vérifie dans le cas du livre d'Astrid Lindgren, pour lequel on peut consulter les dossiers de fabrication à l'IMEC. On peut ainsi lire des notes de lecture indiquant le travail à effectuer pour faire entrer les deux volumes de la traduction de 1951 et 1953 dans le moule de la nouvelle Bibliothèque rose. Il s'agit en partie de formulaires très standardisés avec des cases à cocher.

36 Sur les transformations de la Bibliothèque rose, voir Marc Bauland, Les collections de romans pour la jeunesse de la Librairie Hachette (1945-1980), Mémoire de DEA sous la direction de Jean-Yves Mollier, université de Versailles-Saint-Quentin-en-Yvelines, 1997 et les deux articles de Sophie Heywood déjà cités.

37 Marc Bauland, op. cit., p. 120. Voir aussi « Modernising and moralizing ", op. cit., $\$ 11$. 
Les recommandations portent notamment sur les incorrections lexicales et les répétitions à supprimer, voire même sur le style (la suppression de certains effets, comme les pataquès de Fifi). Les lecteurs mettent en garde contre les attaques politiques ou religieuses, ou encore contre l'utilisation d'un langage aux relents colonialistes. Peut-être moins attendues, certaines propositions portent sur le contenu :

On peut aisément couper. Un certain rajeunissement du style s'impose ça et là. Au début, une légère remise en ordre des événements me semble nécessaire et une atténuation des termes employés par Fifi quand elle se déclare bien heureuse sans ses parents (son père au loin, sa mère morte). Enfin, il y a, page 15, une allusion au Congo belge... Il suffirait de remplacer Congo belge par Afrique. $^{38}$

Ces notes de lecture ne visent donc pas uniquement à communiquer l'appréciation de leur auteur sur l'œuvre, elles proposent aussi des modifications pour la transformer. Les recommandations deviennent d'ailleurs beaucoup plus précises dans un autre commentaire du même lecteur :

Avec " Mademoiselle Brindacier " et "La princesse de Couricoura ", il me semble qu'on peut faire un seul volume de 35000 à 40000 mots. Ce travail est d'autant plus réalisable qu'il ne s'agit pas d'une histoire suivie, mais de chapitres assez indépendants les uns des autres, et dont certains même (d'un volume à l'autre) font presque double emploi.

Un autre lecteur résume bien le malaise que provoque l'anticonformisme de Pippi :

[...] comme dans l'ouvrage précédent, le personnage de Fifi se conforme bien mal aux normes de la Bibliothèque rose. Fifi est une menteuse du commencement à la fin des deux ouvrages. [...] il est de tradition de ne pas ridiculiser les institutions sociales : institutrices, dames d'œuvres, gendarmes, grandes personnes... Fifi, elle, ne s'en prive pas.

Son ambition dans la vie ? " Devenir un tout petit pirate très féroce. Et semer la mort et l'épouvante autour de moi.»

Cette Fifi semble avoir une certaine parenté avec nos blousons noirs... ${ }^{39}$

Il s'agit, on le voit, de passages qui seront supprimés ou transformés dans la deuxième édition ${ }^{40}$. On peut s'étonner du rôle important conféré dans la fabrication d'une œuvre à des personnes qui n'en sont ni l'auteur ni le traducteur. Ce ne sont pas non plus des directeurs de collection et, à vrai dire, on sait fort peu de

38 Dossiers de mise en fabrication, IMEC, HAC 6219 (ex S14 C36 B6), Fiche de lecteur 1 (anonymisé), 29 septembre 1960.

39 Dossiers de mise en fabrication, IMEC, HAC 6219 (ex S14 C36 B6), Fiche de lecteur 2 (anonymisé), 27 septembre 1960.

40 Notons au passage que dans la deuxième édition, Pippi veut seulement "semer l'épouvante " autour d'elle, la référence à la « mort » est supprimée. 
choses de ces lecteurs, si ce n'est que leur rôle est essentiel, puisque leurs recommandations seront suivies, en grande partie, dans la deuxième édition.

S'il n'y a pas de correspondance avec la traductrice dans les archives conservées à l'IMEC et s'il serait intéressant de trouver plus de tapuscrits pour évaluer l'ampleur du processus éditorial, les documents concordent néanmoins pour indiquer des transformations typiques de cette période à la Bibliothèque rose. On peut ainsi se demander si la traductrice, Marie Loewegren, a même collaboré à la deuxième édition. Il existe peu d'informations sur elle. Née Marie-Louise Camus le 20 février 1883 à Paris, elle est morte le 9 octobre 1979 à Stockholm. Lectrice de français au Wellesley college (USA) entre 1906 et 1909, elle a épousé un politicien et historien suédois, Gunnar Löwegrén, le 25 juillet 1909. Tous deux ont eu une activité de traduction : le mari a publié en 1950 (vraisemblablement pendant que Marie-Louise traduisait Astrid Lindgren) une traduction de textes politiques français en suédois ; en plus de la traduction de Pippi Långstrump, la femme a publié celle du livre d'histoire de Nils Ahnlund, Gustave-Adolphe (Paris, Stock, 1936), sous son nom complet (et avec l'orthographe suédoise), MarieLouise Löwegrén.

La traductrice avait 68 ans en 1951, lors de la publication de la première édition de Mademoiselle Brindacier et 79 ans en 1962 au moment de la deuxième édition. Quel rôle a-t-elle pu jouer dans le travail de révision alors qu'elle avait déjà un âge avancé et qu'elle résidait en Suède ? Hachette aurait-il publié la deuxième édition sans la consulter ? Le contrat stipule, au sujet des honoraires de la traductrice : " prix forfaitaire qui nous assurera l'entière propriété de votre texte $»^{41}$. En pensant au droit moral du traducteur, on peut imaginer qu'il devrait être impossible de changer le texte sans son autorisation, comme le garantit la Recommandation de $\mathrm{Nairobi}^{42}$. Dans la pratique, on sait que cela arrive souvent encore aujourd'hui, et que la seule échappatoire pour un traducteur est de refuser que son nom figure sur l'œuvre imprimée ${ }^{43}$.

41 IMEC, Registre copie des contrats, vol. 17, p. 238, 12 mars 1950 et vol. 20, p. 116, $1^{\text {er }}$ juillet 1952.

42 Elle a cependant été publiée en 1976, après la sortie de Fifi Brindacier : « [...] sous réserve des prérogatives de l'auteur de l'auvre préexistante, aucune modification ne sera apportée au texte d'une traduction destinée à la publication sans qu'ait été recherché au préalable l'accord du traducteur [...] " (Recommandation sur la protection juridique des traducteurs et des traductions et sur les moyens pratiques d'améliorer la condition des traducteurs, art. 5, disponible sur http://portal.unesco.org/fr/ev.php-URL_ID=13089\&URL_ DO=DO_TOPIC\&URL_SECTION=201.html)

43 Voir le témoignage de Guillaume Villeneuve, traducteur littéraire : https://www.sgdl.org/ressource/ documentation-sgdl/actes-des-forums/le-droit-moral/1128-le-droit-moral-et-la-traduction-litteraire. Sur la question des droits du traducteur, voir également Salah Basalamah, Le Droit de traduire : une politique culturelle pour la mondialisation, University of Ottawa Press, Les Presses de l'université d'Ottawa, 2009. 
Par ailleurs, l'éditeur a lui aussi un droit et, actuellement, le Code des usages pour la traduction d'une ceuvre de littérature générale précise que lors d'une réédition, «si le traducteur refuse de remanier sa traduction, l'éditeur peut effectuer lui-même le remaniement ou le confier à un tiers " ${ }^{44}$. Il y a donc de fortes chances que, en raison de son âge et de l'éloignement géographique, la traductrice, ne soit pas du tout intervenue sur la deuxième édition et que, comme c'était le cas à l'époque à la Bibliothèque rose, le contrôle éditorial ait été très strict pour conformer le texte aux principes de la collection.

Pippi Långstrump a bel et bien conquis la France, comme le reste du monde, mais cette conquête a connu plusieurs étapes. La première édition, en 1951, présentait une traduction proche du texte suédois qui ne fut pas jugée assez adaptée au marché français en raison du caractère irrévérencieux de l'héroïne, du mauvais exemple qu'elle offrait aux jeunes lecteurs et l'invraisemblance des péripéties décrites dans le livre qui risquaient "de faire confondre le réel et l'imaginaire ${ }^{45}$. Cette traduction a été remaniée pour la deuxième édition, en 1962, qui constitue plutôt une adaptation mettant en avant certains traits de Pippi (force, gentillesse et insouciance), tout en développant le repentir de l'héroïne et en créant des situations où les adultes gardent la maîtrise. Adaptée au format de la Bibliothèque rose, l'œuvre est certes bridée, mais elle connaît alors un plus grand succès dans cette collection où elle prend place aux côtés des best-sellers de l'époque comme Le Club des cinq d'Enid Blyton. Une nouvelle traduction, en 1995, coïncide avec le développement de produits dérivés liés à Pippi Långstrump, comme les livres d'images ou les dessins animés.

Comme le soutient Annelie Jarl Ireman, le lectorat français n'était peut-être pas prêt dans les années 1950-1960 pour l'extrême modernité d'une œuvre comme Pippi Långstrump. Elle n'a d'ailleurs pas vraiment eu le temps de tenter de gagner ce public, puisqu'elle a rapidement été formatée par cette grande machine qu'était la Bibliothèque rose. L'analyse a ainsi montré que l'histoire éditoriale de Mademoiselle Brindacier ne permet pas réellement d'appréhender ce que le lectorat était prêt à accepter ou non, puisque c'est en grande partie la Bibliothèque rose qui a façonné le lectorat français en matière de littérature de jeunesse. Si Pippi Långstrump a conquis la France, dans le sens où elle a ouvert la voie à de nouvelles hérö̈nes et à de nouvelles histoires en littérature jeunesse, on peut au final se demander si ce n'est pas la Bibliothèque rose qui fit véritablement office de conquérant en adaptant l'œuvre à ses propres impératifs socioculturels.

Une réflexion sur le titre français du livre offre à ce sujet un parallèle amusant. Le nom de Fifi Brindacier s'éloigne beaucoup de celui de Pippi Långstrump, alors

44 Texte disponible sur https://www.centrenationaldulivre.fr/fr/ressources/guides-professionnels.

45 Cf. note 27. 
que beaucoup de langues ont conservé un nom proche, ou tout du moins contenant l'image des bas. Le nom de Brindacier semble être une trouvaille tardive : la lettre d'une jeune lectrice de 10 ans à laquelle on avait fait lire en avant-première la traduction du livre d'Astrid Lindgren fait référence à ce dernier sous le titre de Fifi Bradefer (aussi orthographié Brasdefer) ${ }^{46}$. En revanche, le prénom Fifi semble avoir été trouvé plus rapidement. Il n'évoque que peu de références en français et l'héroïne a peu de points communs avec le héros de la nouvelle de Maupassant " Mademoiselle Fifi ». Une autre référence littéraire pourrait cependant offrir une piste intéressante.

Le personnage de Fifi-Dégourdi, créé par Benjamin Rabier et Fred Isly, présente quelques similitudes avec Pippi Långstrump, ou du moins avec la manière dont elle a pu être reçue en France. Fifi-Dégourdi est un enfant «si remuant, si alerte et si éveillé, qu’on l'avait surnommé Fifi-Dégourdi. / Et ce surnom lui allait à merveille, car c'était un diable de petit bonhomme, très espiègle et très malin $»^{47}$. La morale triomphant, on apprend à la fin de l'album qu'une " sévère punition l'avait dégoûté à jamais de tourmenter les autres ». La scène est évoquée sur l'illustration de la couverture, où l'on voit un homme en redingote battre FifiDégourdi à l'aide d'une tapette à tapis, sous l'œil satisfait d'un pompier et d'un policier $^{48}$. Je ne peux m’empêcher de voir cette scène comme une métaphore de l'édition française qui tenterait de faire rentrer dans la norme Pippi Långstrump, ce diable de petite fille, très espiègle et très maligne $e^{49}$.

46 Lettre du 27 octobre 1950 (IMEC, Dossier de fabrication de Fifi Princesse, HAC 6219 - ex S14 C36 B6).

47 Benjamin Rabier et Fred Isly, Une Journée bien remplie, Paris, Félix Juven, 1902.

48 L'album est disponible sur Gallica : https://gallica.bnf.fr/ark:/12148/bpt6k65651993/f1.image. r=fifi-dégourdi.

49 Une liste de titres de travail pour le deuxième volume de la traduction française révèle d'ailleurs une intertextualité avec la comtesse de Ségur : «Un diable de petite fille » (IMEC, Dossier de fabrication de Fifi Princesse, HAC 6219 - ex S14 C36 B6). Ce titre illustre bien le type de réception en France de Pippi Långstrump, dont on voulait condamner le comportement. 